\title{
Transporte cicloviário no ambiente urbano: síntese do conhecimento no período de
}

\section{0-2019}

\author{
Cycling transport in the urban environment: synthesis of knowledge in the period 1990-2019 \\ Transporte en bicicleta en el entorno urbano: síntesis de conocimientos en el período 1990-2019
}

Recebido: 19/08/2021 | Revisado: 25/08/2021 | Aceito: 30/08/2021 | Publicado: 01/09/2021

Cláudia Sabrina Pereira Lopes

ORCID: https://orcid.org/0000-0002-1751-4935

Universidade Federal de Mato Grosso do Sul, Brasil

E-mail: csabrinaplopes@gmail.com

Jaíza Santos Motta

ORCID: https://orcid.org/0000-0002-3714-6631

Universidade Federal de Mato Grosso do Sul, Brasil

E-mail: ea.jsmotta@gmail.com

Antonio Conceição Paranhos Filho

ORCID: https://orcid.org/0000-0002-9838-5337 Universidade Federal de Mato Grosso do Sul, Brasil

E-mail: toniparanhos@gmail.com

Eliane Guaraldo

ORCID: https://orcid.org/0000-0003-2526-1293

Universidade Federal de Mato Grosso do Sul, Brasil

E-mail: eliane.guaraldo@gmail.com

\begin{abstract}
Resumo
$\mathrm{O}$ uso da bicicleta nas cidades tem crescido em todo o mundo em virtude das políticas de mobilidade urbana sustentável. Este artigo tem por finalidade analisar as tendências e a produção científica de pesquisa sobre a expansão da utilização de bicicleta no ambiente urbano entre 1990 e 2019. Foi empregada a bibliometria, com alvo de análise em tipos de publicações, modalidade de acesso, idiomas das publicações, características dos artigos, periódicos proeminentes, autores em destaque, palavras-chave e países das publicações. Um software de elaboração mapas bibliométricos foi empregado para visualização gráfica de ocorrência simultânea de palavras-chave e de acoplamento bibliográfico entre países. Foram encontradas 1559 publicações no Scopus, abrangendo 14 diferentes tipos de documentos. Grande parte das publicações foram escritas em inglês $(96,34 \%)$ e possuíam acesso restrito $(75,37 \%)$. A classe Artigos de Pesquisa destacou-se com maior parcela da produção $(68,44 \%)$. Houve uma tendência constante de crescimento na quantidade de publicações ao ano. As palavras-chave identificadas estão vinculadas às temáticas de Transporte Ativo, Saúde e Planejamento Urbano. Estados Unidos, Austrália, Canadá, Bélgica e Reino Unido foram os países que mais se destacaram por apresentar referências em comum entre os artigos.
\end{abstract}

Palavras-chave: Ciclismo; Mobilidade ativa; Planejamento territorial.

\begin{abstract}
Cycling use in cities has grown around the world as a result of sustainable urban mobility policies. This article aims to analyze trends and scientific research production on the expansion of bicycle use in the urban environment between 1990 and 2019. Bibliometrics was used aiming to analysing types of publications, access modality, publication languages, characteristics of articles, prominent journals, featured authors, keywords and countries of publications. A bibliometric map elaboration software was used for graphic visualization of simultaneous occurrence of keywords and bibliographic coupling between countries. 1559 publications were found in Scopus, covering 14 different types of documents. Most publications were written in English (96.34\%) and had restricted access (75.37\%). The Research Articles class stood out with the largest share of production (68.44\%). There was a constant growth trend in the number of publications per year. The identified keywords are linked to the themes of Active Transport, Health and Urban Planning. The United States, Australia, Canada, Belgium and the United Kingdom were the countries that most stood out for having common references among the articles.
\end{abstract}

Keywords: Cycling; Active mobility; Territorial planning.

\section{Resumen}

El uso de la bicicleta en las ciudades ha crecido en todo el mundo como resultado de las políticas de movilidad urbana sostenible. Este artículo tiene como objetivo analizar las tendencias y la producción de investigación científica sobre la expansión del uso de la bicicleta en el medio urbano entre 1990 y 2019. Se utilizó la bibliometría, con el objetivo de analizar en tipos de publicaciones, modalidad de acceso, lenguajes de publicación, características de los artículos, 
destacados revistas, autores destacados, palabras clave y países de publicación. Se utilizó un software de elaboración de mapas bibliométricos para la visualización gráfica de la ocurrencia simultánea de palabras clave y acoplamiento bibliográfico entre países. Se encontraron 1559 publicaciones en Scopus, que cubren 14 tipos diferentes de documentos. La mayoría de las publicaciones estaban escritas en inglés (96,34\%) y tenían acceso restringido (75,37\%). La clase de Artículos de investigación se destacó con la mayor participación de la producción $(68,44 \%)$. Hubo una tendencia de crecimiento constante en el número de publicaciones por año. Las palabras clave identificadas están vinculadas a los temas de Transporte Activo, Salud y Urbanismo. Estados Unidos, Australia, Canadá, Bélgica y Reino Unido fueron los países que más destacaron por tener referencias comunes entre los artículos.

Palabras clave: Ciclismo; Movilidad activa; Planificación territorial.

\section{Introdução}

O planejamento de transportes e o modo como o solo urbano está organizado são elementos profundamente interligados. Contudo, é notada uma carência de estudos relacionando as características de transporte e as classes de uso e cobertura do solo. Ademais, é percebida pouca ênfase na produção científica sobre as consequências da integração de infraestrutura para bicicletas, pedestres e veículos motorizados (Wang, et al., 2019).

$\mathrm{O}$ uso de bicicleta tem sido incentivado em cidades de todo o mundo devido aos benefícios à saúde física e mental, à redução de emissão de poluentes atmosféricos e à economia de espaço (Götschi et al., 2018; Wang, Y., et al., 2019), entre outras razões pelo fato de que esta modalidade de transporte é considerada sustentável quando comparada aos veículos motorizados (Gatersleben et al., 2007; Wang, et al., 2019).

A utilização da bicicleta auxilia no desempenho pulmonar aeróbio e contribui para obtenção dos níveis considerados adequados para função pulmonar (Berrigan et al., 2006; De Geus et al., 2007; Dill, 2009; Wang, et al., 2019). Ademais, seu uso com frequência pode combater o modo de vida sedentário dos habitantes (Haskell, 2004; Titze et al., 2008; Wang, et al., 2019).

Além das já reconhecidas contribuições para a saúde, a bicicleta estimula a atividade física (Ma \& Dill, 2015; Sallis et al., 2004; Wang, et al., 2019). Os exercícios físicos, por sua vez, estão comprovadamente associados à diminuição do risco de doenças cardiovasculares, diabetes e cânceres de cólon, mama e endometrial (Moore et al., 2016; Nocon et al., 2008; Wang, et al., 2019).

A importância e o uso crescente de bicicletas em meio urbano vem atraindo a atenção da comunidade científica global para esta temática. A Bibliometria, campo de conhecimento que oferece metodologias objetivas para avaliar a relevância de um artigo para o progresso da ciência (Huang \& Zhao, 2008; Yang et al., 2013), foi utilizada neste estudo. Este artigo tem por objetivo examinar o status atual e tendências de pesquisa de uso da bicicleta no ambiente urbano no período de 30 anos compreendido de 1990 a 2019 para permitir o entendimento da dinâmica mundial de pesquisas sobre o ciclismo em cidades e das perspectivas futuras de publicações para o tema.

\section{Metodologia}

\subsection{Seleção da fonte de dados}

As bases científicas mais amplamente empregadas para aquisição de informações bibliométricas são a Google Scholar, a Web of Science (WoS) e a Scopus (Kaur \& Sood, 2019).

A WoS (https://www-webofscience.ez51.periodicos.capes.gov.br/wos/woscc/basic-search) é considerada uma base científica ímpar (Kaur \& Sood, 2019). Esta base dispõe de 3 índices de citação: Science Citation Index Expanded; Social Sciences Citation Index; e Arts\&Humanities Citation Index (Kaur \& Sood, 2019; Norris \& Oppeheim, 2007).

A Google Scholar (https://scholar.google.com.br/schhp?hl=pt-BR\&as_sdt=0,5) possibilita acessar gratuitamente material acadêmico, em contraponto com as outras 2 bases mencionadas, e assim impulsiona as buscas por publicações científicas (Kaur \& Sood, 2019; Norris \& Oppeheim, 2007). Entretanto, possui falhas que prejudicam a confiabilidade dos 
dados apresentados, como por exemplo, a contagem equivocada de citações (Kaur \& Sood, 2019; Jacso, 2006).

Para efetuar o estudo bibliométrico, foi, inicialmente, executada uma pesquisa preliminar nas bases de dados científicas Scopus e WoS. Entre a Scopus e a WoS, a Scopus (https://www-scopus.ez51.periodicos.ca pes.gov.br/search/form.uri?display=basic\#basic) é mais vantajosa, visto que cobre uma quantidade maior e mais atualizada de publicações com qualidade diferenciada, além de conter um exame detalhado das citações (Kaur \& Sood, 2019; Meho \& Rogers, 2008), notadamente no que se refere às Ciências. Farrukh et al. (2020) também apontam a Scopus como uma base de dados mais completa do que a WoS.

\subsection{Definição do termo de busca (TB)}

Para assegurar que o mecanismo de busca encontrasse o máximo possível de publicações científicas relacionadas ao uso de bicicleta no ambiente urbano, ou seja, de forma abrangente, considerou-se como Termo de Busca (TB) a expressão ("bicycl*” OR "bike*” OR “cycling”) AND ("urban planning” OR “urban mobility” OR "built environment"), para o intervalo temporal de 1990 a 2019.Esse TB foi procurado nos campos Título da Publicação, Resumo e Palavras-Chave. Cabe salientar que o caractere asterisco (*) representa um operador de truncamento empregado para encontrar plurais e variações grafias de zero a infinitos caracteres.

\subsection{Tendências em pesquisa avaliadas}

Com os metadados coletados do Scopus, a pesquisa foi direcionada a verificar as tendências e performance dos resultados no âmbito do uso da bicicleta no meio urbano. Foram apreciados os seguintes parâmetros bibliométricos: tipo e o idioma das publicações, a característica de saída dos artigos, periódicos proeminentes e principais autores. A escolha destes critérios de avaliação foi baseada nos trabalhos de Yang et al. (2013) e Milaré et al., (2016).

\subsection{VOS Viewer como ferramenta de análise bibliométrica}

Mecanismos de visualização de dados são úteis à compreensão de padrões e correlações de dados organizados em extensas quantidades de texto e que por este motivo não ficam tão claros (Kaur \& Sood, 2019). Entre estes mecanismos, se destacam o Gephi, o VOS Viewer e o Cite Space (Kaur \& Sood, 2019).

Neste estudo foi utilizado o software gratuito VOS Viewer (Van Eck \& Waltman, 2010), empregado para a transformação de dados bibliométricos em gráficos ilustrativos (Farrukh et al., 2020). Nesta pesquisa, os parâmetros bibliométricos a serem avaliados foram ocorrência simultânea de palavras-chaves de autores e acoplamento bibliográfico entre países.

O acoplamento bibliográfico acontece no contexto em que duas publicações citam uma terceira (Farrukh et al., 2020; Kessler, 1963). Já a ocorrência simultânea de palavras-chave verifica as palavras-chave mais reportadas dentro da amostra de documentos científicos em estudo (Farrukh et al., 2020).

O VOS Viewer (Van Eck \& Waltman, 2010) permite identificar a frequência com que certos termos ocorrem em rede, além da frequência com a qual ocorrem juntos.

O programa também possibilita o agrupamento de dados em Clusters e, em consequência, a diferenciação das áreas de pesquisa do tema estudado. Esse programa reúne recursos fundamentais para visualização e possui uma poderosa interface gráfica ideal para explorar e estudar diversas redes (Cobo et al., 2011; Eck \& Waltman, 2010; Kaur \& Sood, 2019). 


\section{Resultados e Discussão}

\subsection{Escolha da fonte de dados}

A pesquisa na base WoS retornou 1.239 resultados, ao passo em que foram obtidos 1.559 resultados para a Scopus. Logo, a Scopus encontrou mais trabalhos quando comparada a WoS. Desta forma, optou-se por realizar a análise bibliométrica com os metadados adquiridos no Scopus.

\subsection{Tipos de publicações, modalidades de acesso e idioma das publicações}

A partir do TB escolhido, foram encontradas 1.559 publicações no Scopus, abrangendo 14 diferentes classes de documentos. (Figura 1). Do total, 1067 constituem Artigos de Pesquisa, o que corresponde a 68,44\% da produção integral. Em seguida, estão as seguintes categorias: Artigos de Conferência (343;22\%); Artigos de Revisão (68;4,36\%); Capítulos de Livro (41;2,63\%); Artigos de Revisão de Conferência (17;1,09\%); Livros (12;0,77\%); Short Survey (3;0,19\%); Notas (2;0,13\%); Editorial(1;0,06\%); Data Paper (1;0,06\%); Errata(1;0,06\%); Carta $(1 ; 0,06 \%)$; e Relatório (1;0,06\%). Não foi possível determinar a natureza de apenas 1 dos resultados, sendo este classificado como "Indefinido" (1;0,06\%). Dessa forma, como os Artigos de Pesquisa revelaram ser a categoria mais expressiva em quantidade de publicações foi esta a categoria de publicação escolhida para foco das análises.

Figura 1 - Representação gráfica dos tipos de publicação encontrados para o TB estudado.

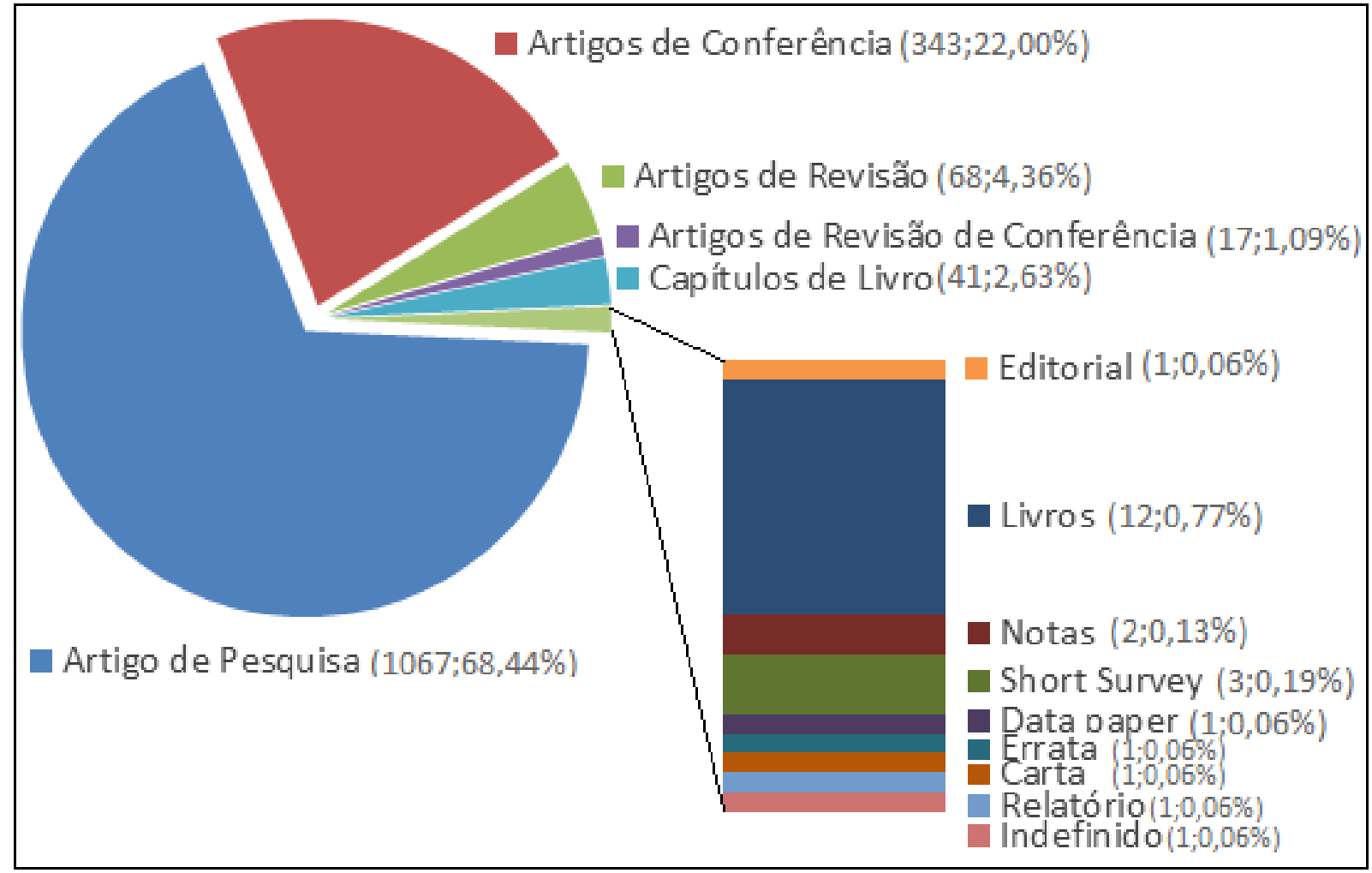

Fonte: Autores. 
Quanto à modalidade de acesso, foi verificado que apenas 384 publicações (34,63\%) apresentam acesso aberto. Os demais 1175 documentos (75,37\%) apresentam restrição de acesso (Figura 2).

Figura 2 - Representação gráfica das publicações relação à modalidade de acesso.

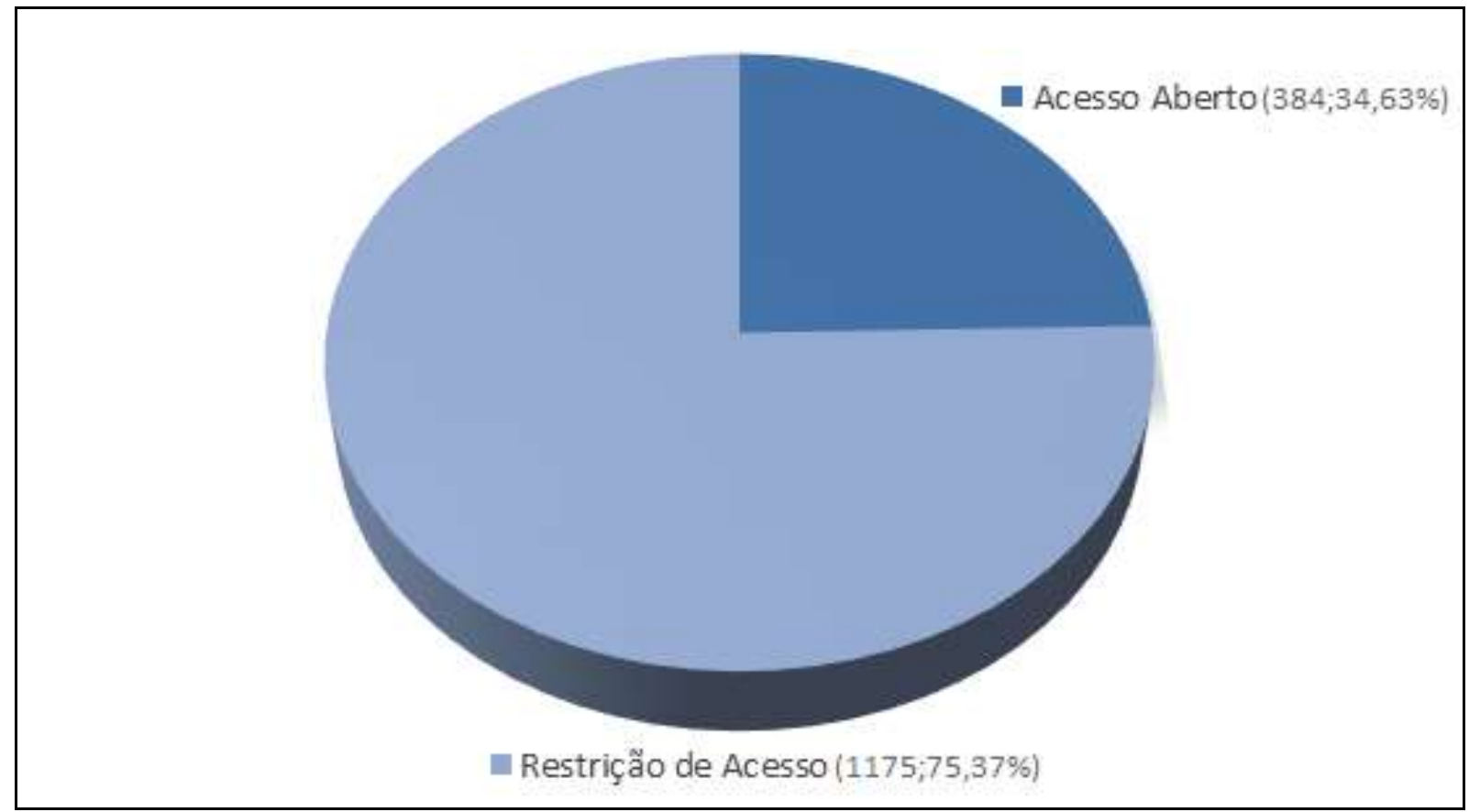

Fonte: Autores.

Acerca do idioma, foi observado que quase a totalidade dos documentos foi escrita em língua inglesa (1502; 96,34\%), conforme é possível observar na Figura 3. Contudo, cabe salientar a diversidade de idiomas que aparecem, ainda que com quantidades de publicações não tão expressivas. Além do Inglês, foram identificados documentos em outros 13 idiomas: Chinês (12;0,77\%); Português (10;0,64\%); Espanhol (8;0,51\%); Francês $(8 ; 0,51 \%)$; Japonês $(3 ; 0,19 \%)$; Alemão $(2 ; 0,13 \%)$; Italiano (2;0,13\%); Finlandês ( $1 ; 0,06 \%)$, Húngaro (1;0,06\%); Persa (1;0,06\%); Russo (1;0,06\%); Sérvio (1;0,06\%); e Esloveno $(1 ; 0,06 \%)$.

Foi constatada também a situação em que 5 documentos estavam disponíveis em mais de um idioma $(0,32 \%)$, sendo o Inglês uma dessas línguas. Não foi possível determinar o idioma de 1 publicação, sendo este classificado como "Desconhecido" para fins deste estudo. 
Figura 3 - Representação gráfica das publicações por idioma.

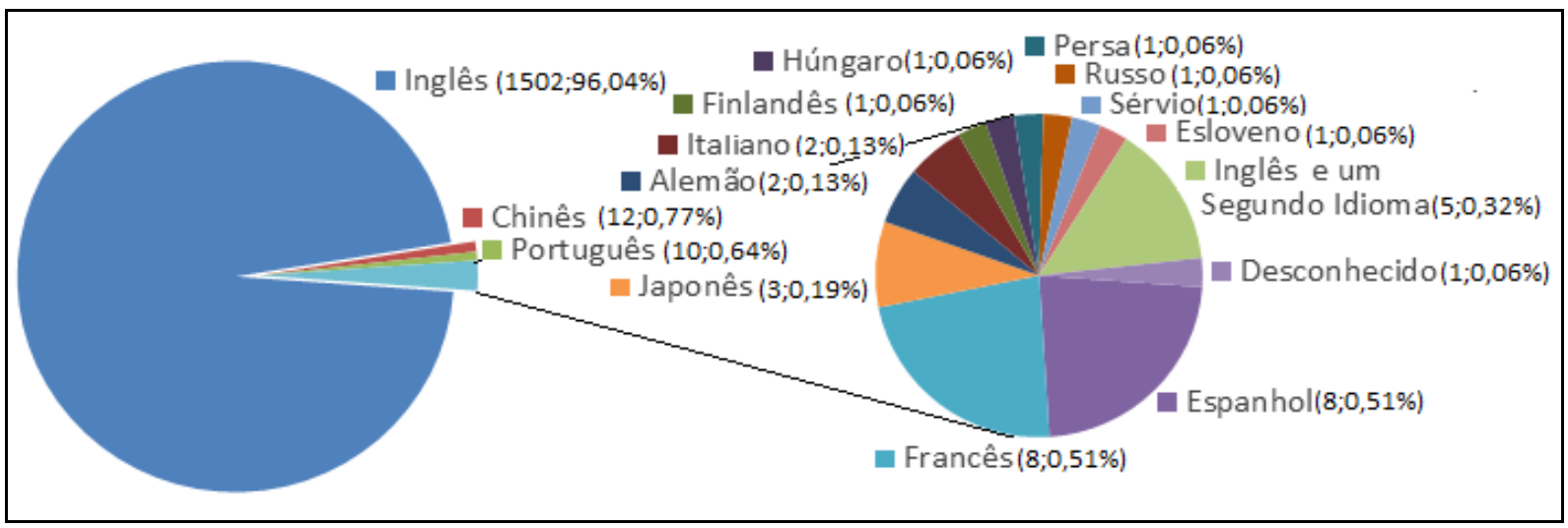

Fonte: Autores.

\subsection{Análise de características de produção dos artigos}

O número de artigos ano a ano sofreu oscilações positivas e negativas, porém, avaliando-se o intervalo total de 30 anos, infere-se que há um padrão de crescimento de 1990 a 2019. A quantidade artigos ampliou de 2 em 1990 para $182 \mathrm{em}$ 2019 (Figura 4).

Figura 4 - Evolução do número de artigos publicados sobre o uso de bicicleta no ambiente urbano durante 1990-2019.

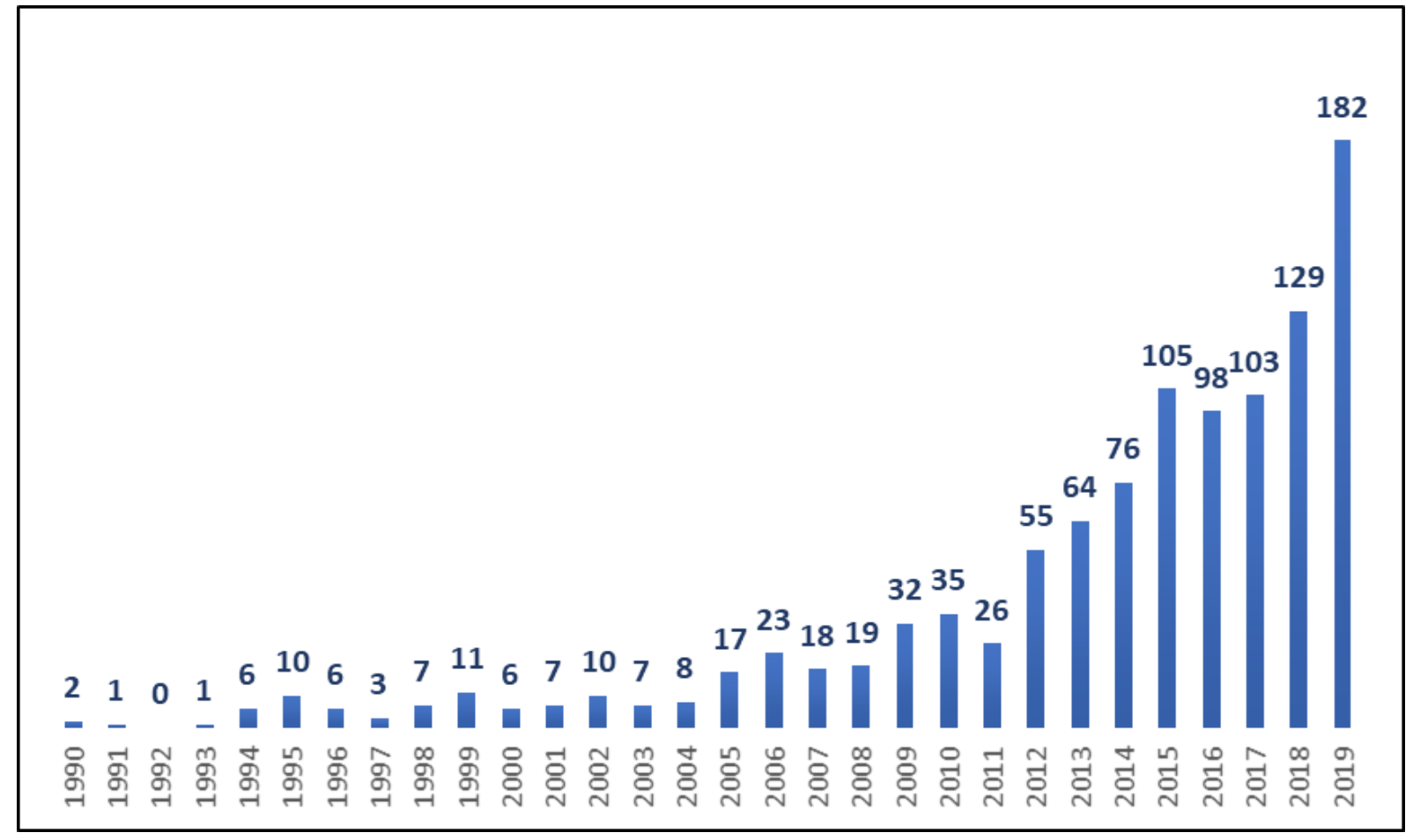

Fonte: Autores.

\subsection{Periódicos proeminentes}

A Tabela 1 apresenta os periódicos que publicaram 10 ou mais artigos sobre o assunto dentro do intervalo de 1990 a 2019. Há 534 dos 1067 artigos distribuídos em 26 periódicos. Este valor demonstra que em torno de 50,05\% do total de artigos estão concentrados nestas revistas.

Entre os 26 periódicos listados, Transportation Research Record (https://journals.sagepub.com/home/trr), Journal Of Transport Geography (https://www.journals.elsevier.com/journal-of-transport-geography) e Journal Of Transport and Health 
(https://www.journals.elsevier.com/journal-of-transport-and-health) foram os que mais se destacaram em termos quantitativos nos 30 anos.

Logo, infere-se que, ao se estudar o uso da bicicleta em meio urbano, há uma tendência em integrar o planejamento de transportes com políticas públicas de saúde, prevenção de acidentes e sustentabilidade.

Tabela 1 - Periódicos que publicaram 10 ou mais artigos sobre o assunto durante 1990-2019.

\begin{tabular}{|c|c|c|c|}
\hline ID & Nome do Periódico & Total de Artigos & Total de Citações no Scopus \\
\hline 1 & Transportation Research Record & 68 & 1570 \\
\hline 2 & Journal of Transport Geography & 41 & 1116 \\
\hline 3 & Journal of Transport and Health & 33 & 476 \\
\hline 4 & Accident Analysis And Prevention & 29 & 511 \\
\hline 5 & International Journal of Behavioral Nutrition and Physical Activity & 26 & 756 \\
\hline 6 & Sustainability Switzerland & 26 & 155 \\
\hline 7 & Transportation Research Part A Policy and Pratice & 25 & 1465 \\
\hline 8 & Transportation Research Part D Transport and Environment & 24 & 851 \\
\hline 9 & Preventive Medicine & 20 & 584 \\
\hline 10 & American Journal of Preventive Medicine & 19 & 1633 \\
\hline 11 & International Journal of Environmental Research and Public Health & 18 & 203 \\
\hline 12 & Journal of Physical Activity and Health & 19 & 258 \\
\hline 13 & Landscape And Urban Planning & 18 & 639 \\
\hline 14 & Planning & 16 & 7 \\
\hline 15 & BMC Public Health & 15 & 202 \\
\hline 16 & Health and Place & 15 & 490 \\
\hline 17 & Journal Of Transport and Land Use & 14 & 164 \\
\hline 18 & Transportation & 14 & 530 \\
\hline 19 & International Journal of Sustainable Transportation & 13 & 568 \\
\hline 20 & Transport Policy & 13 & 368 \\
\hline 21 & American Journal of Health Promotion & 13 & 263 \\
\hline 22 & Cities & 12 & 244 \\
\hline 23 & Journal of Urban Planning and Development & 12 & 477 \\
\hline 24 & Journal of Urban Health & 11 & 512 \\
\hline 25 & Proceedings of The Institution Os Civil Engineers Municipal Engineer & 10 & 26 \\
\hline 26 & Wit Transactions on Ecology and the Environment & 10 & 12 \\
\hline
\end{tabular}

Fonte: Autores.

\subsection{Autores em destaque}

A Tabela 2 apresenta os autores que publicaram 10 ou mais artigos sobre o assunto dentro do intervalo de 1990 a 2019. Há 204 dos 1067 artigos distribuídos entre 14 autores. Este valor demonstra que em torno de 19,12\% do total de artigos estão concentrados nesses autores. Os pesquisadores que mais publicaram artigos foram De Bourdeaudhuij, I. (26), Sallis J. F. (26) e Deforche B. (18). 
Tabela 2 - Autores que publicaram 10 ou mais artigos sobre o assunto no intervalo de 1990-2019.

\begin{tabular}{|c|c|c|c|}
\hline ID & Nome do Autor & Afiliação & $\begin{array}{c}\text { Quantidade de } \\
\text { Artigos Relacionados } \\
\text { ao TB } \\
\end{array}$ \\
\hline 1 & De Bourdeaudhuij, I. & Universiteit Gent (BEL) & 26 \\
\hline 2 & Sallis J. F. & Australian Catholic University (AUS) & 26 \\
\hline 3 & Deforche B. & Universiteit Gent (BEL) & 18 \\
\hline 4 & Van Dyck, D. & Universiteit Gent (BEL) & 15 \\
\hline 5 & Conway T.L. & $\begin{array}{l}\text { Department of Family Medicine and Public Health } \\
\text { (USA) }\end{array}$ & 14 \\
\hline 6 & Frank, L. D. & University Of British Columbia (CAN) & 14 \\
\hline 7 & Giles-Corti, B. & RMIT University (AUS) & 14 \\
\hline 8 & Kerr, J. & $\begin{array}{l}\text { Department of Family Medicine and Public Health } \\
\text { (USA) }\end{array}$ & 14 \\
\hline 9 & Saelens, B.E. & University of Washington (USA) & 13 \\
\hline 10 & Brownson, R.C. & Washington University in St Louis (USA) & 10 \\
\hline 11 & Cardon, G. & University of Ghent (BEL) & 10 \\
\hline 12 & Cerin, E. & Australian Catholic University (AUS) & 10 \\
\hline 13 & Chen, $\mathrm{P}$. & Tongji University (CHN) & 10 \\
\hline 14 & Winters, M. & University of British Columbia (CAN) & 10 \\
\hline
\end{tabular}

Fonte: Autores.

\subsection{Análise no software VOS Viewer}

Apenas o estrato "Artigos de Pesquisa" foi analisado no software VOS Viewer (Van Eck \& Waltman, 2010), por ser a categoria de publicações mais expressiva.

\section{a) Ocorrência simultânea de palavras-chave de autores}

A ocorrência simultânea expressa o quanto uma palavra-chave aparece junto a outras palavras-chave (Farrukh et al., 2020). Analisar a ocorrência simultânea de palavras-chave é pertinente, pois revela um panorama do quê tem sido publicado sobre o assunto, além de apontar perspectivas para trabalhos futuros, isto é, como tende a ser a produção científica em determinado contexto (Farrukh et al., 2020). De acordo com as estatísticas do software, no total foram encontradas 2.342 palavras-chave.

Foi adotado um valor mínimo de 12 ocorrências para a geração do mapa gráfico e assim, foram selecionadas 35 palavras-chave pelo programa. Optou-se por essa quantidade palavras-chave para que o mapa gráfico abrangesse apenas as 35 palavras-chave mais relevantes de modo a otimizar a visualização das palavras exibidas e evitar possíveis sobreposições ou poluição visual.

Na Tabela 3 são exibidas as 35 palavras-chave mais reportadas para o TB adotado, considerando o estrato Artigos, de 1990 a 2019. Infere-se que a maior parcela das palavras-chave está relacionada ao Transporte Ativo, Saúde e Planejamento Urbano. 
Research, Society and Development, v. 10, n. 11, e283101119616, 2021

(CC BY 4.0) | ISSN 2525-3409 | DOI: http://dx.doi.org/10.33448/rsd-v10i11.19616

Tabela 3 - Palavras-chave de autores mais utilizadas no período de 1990-2019.

\begin{tabular}{|c|c|c|c|}
\hline ID & Palavra-chave & Número de Ocorrências & Força Total de Ligação \\
\hline 1 & Built environment & 228 & 350 \\
\hline 2 & Physical activity & 125 & 242 \\
\hline 3 & Walking & 56 & 156 \\
\hline 4 & Cycling & 80 & 149 \\
\hline 5 & Bicycling & 44 & 93 \\
\hline 6 & Walkability & 31 & 77 \\
\hline 7 & GIS & 28 & 65 \\
\hline 8 & Active transportation & 28 & 64 \\
\hline 9 & Active travel & 27 & 52 \\
\hline 10 & Neighborhood & 17 & 50 \\
\hline 11 & Urban Planning & 42 & 48 \\
\hline 12 & Active Transport & 21 & 45 \\
\hline 13 & Travel Behavior & 22 & 42 \\
\hline 14 & Infrastructure & 18 & 41 \\
\hline 15 & Transport & 24 & 41 \\
\hline 16 & Health & 18 & 38 \\
\hline 17 & Bicycle & 29 & 37 \\
\hline 18 & Transportation & 23 & 37 \\
\hline 19 & Policy & 18 & 36 \\
\hline 20 & Urban Design & 14 & 35 \\
\hline 21 & Children & 14 & 34 \\
\hline 22 & Obesity & 18 & 34 \\
\hline 23 & Active Living & 13 & 33 \\
\hline 24 & Urban Form & 12 & 31 \\
\hline 25 & Public Health & 17 & 30 \\
\hline 26 & Exercise & 15 & 28 \\
\hline 27 & China & 22 & 27 \\
\hline 28 & Environment & 17 & 23 \\
\hline 29 & Mode Choice & 17 & 20 \\
\hline 30 & Sustainability & 15 & 19 \\
\hline 31 & Mobility & 18 & 17 \\
\hline 32 & Urban Mobility & 37 & 13 \\
\hline 33 & Acessibility & 15 & 12 \\
\hline 34 & Sustainable Mobility & 12 & 11 \\
\hline 35 & Bicycles & 12 & 10 \\
\hline
\end{tabular}

Fonte: Autores.

A Figura 5 evidencia as conexões entre as palavras-chave. No software VOS Viewer (Van Eck \& Waltman, 2010) foi criada uma rede, destacando a frequência com que cada termo apareceu nos artigos avaliados, e ainda, a frequência em que esses termos apareceram interligados entre si. Cada círculo está associado a uma palavra-chave. O tamanho deste e da fonte da palavra-chave é proporcional a frequência de ocorrência. A rede construída pelo software exibiu a reunião das palavras-chave em Clusters, cuja distinção foi possível através das cores. 
As palavras-chave agrupadas em um mesmo Cluster ocorrem juntas com uma maior frequência, situação que não impossibilita associação com elementos de outros Clusters (Glińska \& Siemieniako, 2017).

Figura 5 - Representação gráfica da ocorrência simultânea das palavras-chave dos autores, elaborada no software VOS Viewer (Van Eck \& Waltman, 2010). Como parâmetro de entrada, foi adotado um valor mínimo de 12 ocorrências para a geração do mapa gráfico e assim, do total de 2.342 palavras-chave encontradas foram selecionadas 35 palavras-chave mais relevantes.

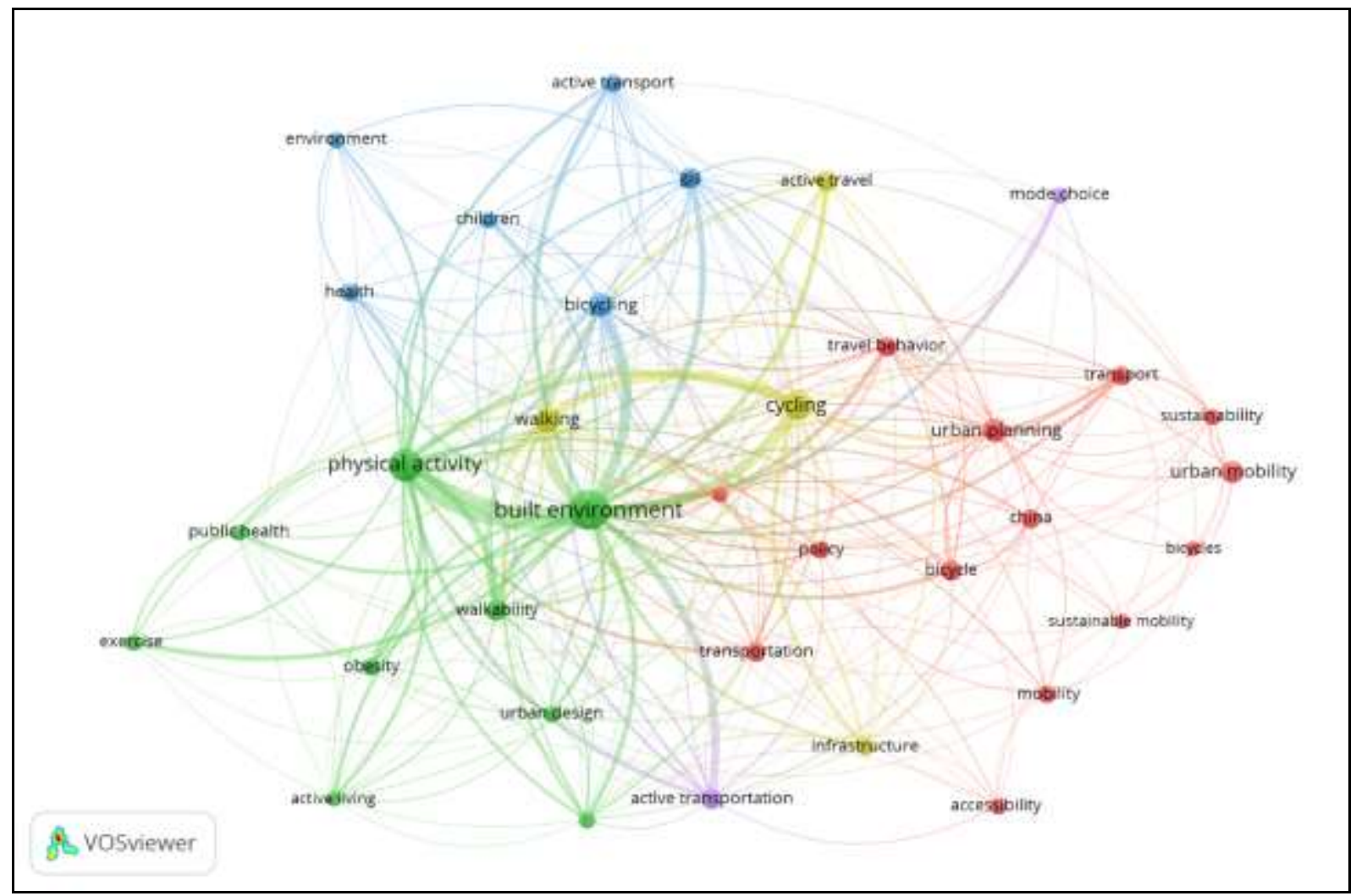

Fonte: Autores.

Para facilitar a compreensão do agrupamento de palavras, foram propostos nomes para os Clusters considerando a maioria das respectivas palavras associadas a eles, procedimento observado no estudo de Glińska e Siemieniako (2017). Assim, foram selecionadas 35 palavras-chave e identificados 5 Clusters conforme caracterizado a seguir.

- Cluster 1 (Azul). O Ciclismo e sua interação com o Ambiente: Environment, children, bicycling, health, active transport, gis;

- Cluster 2 (Amarelo). Relação entre Infraestrutura e Viagens Ativas. Infrastructure, cycling, walking, active travel;

- Cluster 3 (Lilás). Modos de Transporte. Mode choice, active transportation;

- Cluster 4 (Vermelho). A Bicicleta como Objeto Propulsor de Sustentabilidade. Travel behaviour, urban planning, transport, sustainability, urban mobility, china, policy, bicycles, bicycle, sustainable mobility, accessibility, transportation, urban form;

- Cluster 5 (Verde). O Desenho Urbano e sua relação com a Saúde Pública. Built environment, physical activity, walkability, public health, exercise, obesity, active living, urban design, neighborhood. 


\section{b) Acoplamento bibliográfico entre países}

Outro parâmetro bibliométrico analisado foi o acoplamento bibliográfico entre países que publicaram e foram encontrados através do Termo de Busca (TB). A conexão entre países é estabelecida considerando o número de referências em comum entre os artigos. De acordo com as estatísticas do software, no total, foram encontrados 73 países.

Para a análise, foi estipulado o valor 7 como número mínimo de documentos por país. Destarte, foram selecionados 29 países, distribuídos em 3 Clusters. De forma análoga à análise de ocorrência simultânea de palavras-chave, optou-se por esta quantidade de documentos por país para que o mapa gráfico abrangesse apenas os 29 países mais relevantes, de modo a otimizar a visualização e evitar possíveis sobreposições ou poluição visual.

A Tabela 4 contém os 29 países selecionados e demonstra as relações entre o número de artigos, citações e força total de ligação entre os países.

Tabela 4 - Países com maiores índices de acoplamento bibliográfico considerando a força total de ligação entre si.

\begin{tabular}{|c|c|c|c|c|}
\hline ID & País & $\mathbf{N}^{\circ}$ de Artigos & Citações & $\begin{array}{c}\text { Força Total de } \\
\text { Ligação }\end{array}$ \\
\hline 1 & Estados Unidos & 409 & 14204 & 114634 \\
\hline 2 & Austrália & 111 & 3054 & 55063 \\
\hline 3 & Canadá & 125 & 4049 & 53964 \\
\hline 4 & Bélgica & 47 & 1534 & 36014 \\
\hline 5 & Reino Unido & 104 & 2988 & 34758 \\
\hline 6 & China & 108 & 1860 & 32058 \\
\hline 7 & Hong Kong & 26 & 1130 & 26285 \\
\hline 8 & Holanda & 48 & 1028 & 20018 \\
\hline 9 & Nova Zelândia & 26 & 1230 & 18738 \\
\hline 10 & Brasil & 35 & 579 & 16655 \\
\hline 11 & Espanha & 37 & 641 & 15880 \\
\hline 12 & Colômbia & 19 & 929 & 14794 \\
\hline 13 & Dinamarca & 22 & 647 & 13351 \\
\hline 14 & França & 22 & 448 & 11300 \\
\hline 15 & Japão & 20 & 322 & 9596 \\
\hline 16 & República Tcheca & 13 & 363 & 8678 \\
\hline 17 & Suécia & 22 & 411 & 8539 \\
\hline 18 & Alemanha & 35 & 634 & 7851 \\
\hline 19 & México & 9 & 246 & 7709 \\
\hline 20 & Hungria & 12 & 217 & 7536 \\
\hline 21 & Noruega & 14 & 160 & 6980 \\
\hline 22 & Áustria & 15 & 394 & 6363 \\
\hline 23 & Finlândia & 14 & 317 & 5484 \\
\hline 24 & Chile & 17 & 199 & 4340 \\
\hline 25 & Itália & 28 & 225 & 3210 \\
\hline 26 & Coréia do Sul & 12 & 101 & 2776 \\
\hline 27 & Cingapura & 11 & 179 & 2628 \\
\hline 28 & Suíça & 13 & 371 & 2608 \\
\hline 29 & Portugal & 10 & 50 & 2550 \\
\hline
\end{tabular}


A Figura 6 evidencia o acoplamento bibliográfico entre países, sendo possível observar os 3 Clusters. O Cluster 1 (Azul) tem como liderança os Estados Unidos, o qual está diretamente vinculado às pesquisas executadas pela China, Canadá, Alemanha, Coréia do Sul, Chile e Singapura.

Já no Cluster 2 (Verde) o país que mais se destaca em termos de publicações é a Austrália, a qual se conecta de forma direta com o Japão, Hong Kong, México, Colômbia, Brasil, Dinamarca, República Tcheca, Noruega, Nova Zelândia e Bélgica.

Por fim, o Cluster 3 (Vermelho) é liderado pelo Reino Unido, o qual tem conexão direta com pesquisas da Suécia, Espanha, Finlândia, Áustria, Holanda, Itália, Portugal, França, Hungria e Suíça. Especialmente neste agrupamento, nota-se relações diretas em pesquisa de países pertencentes a um mesmo continente.

Figura 6 - Representação gráfica do acoplamento bibliográfico entre países., elaborada no software VOS Viewer (Van Eck \& Waltman, 2010).

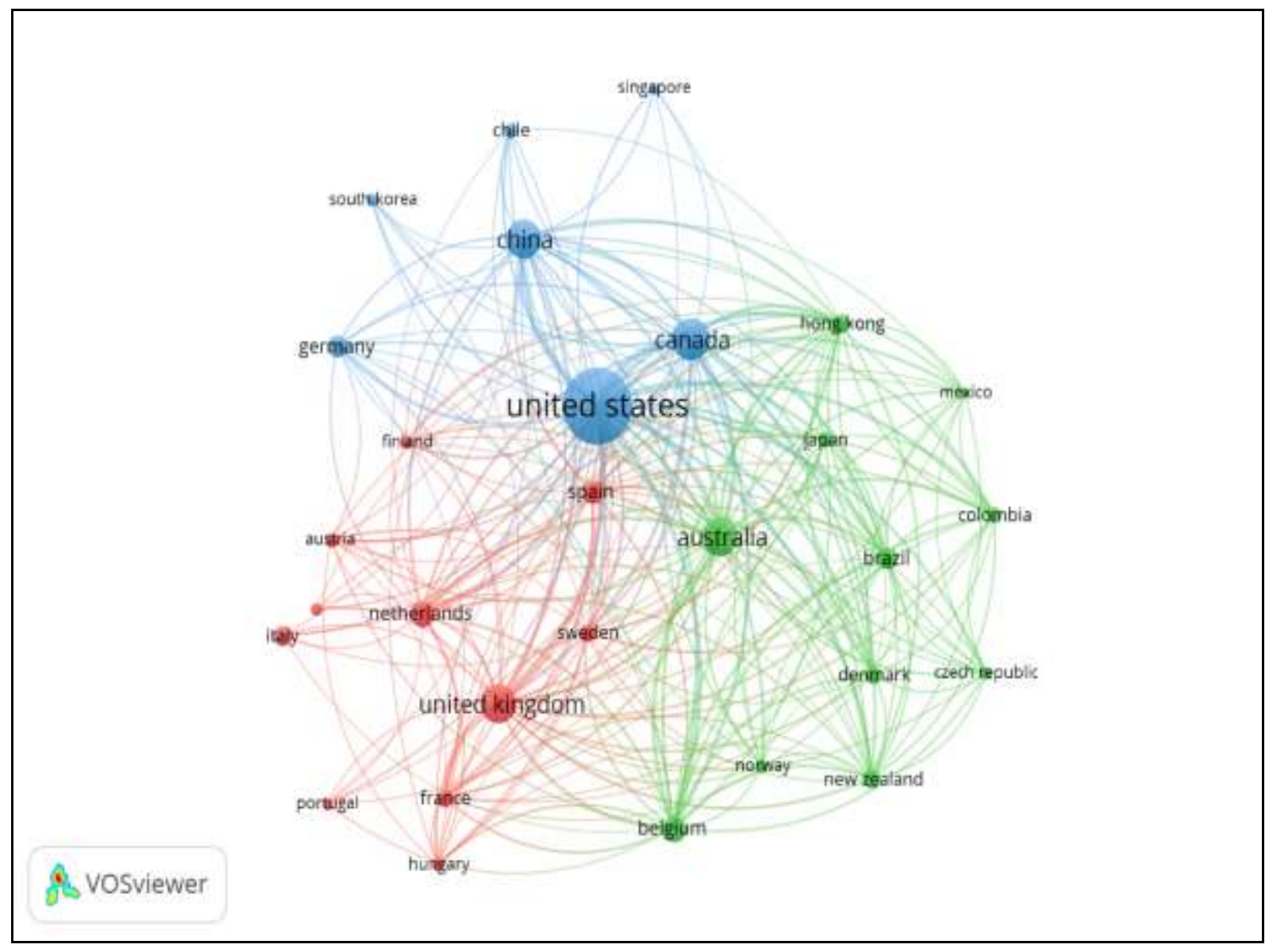

Fonte: Autores.

\section{Considerações Finais}

Este estudo revelou pontos significativos sobre as tendências de pesquisa para o uso da bicicleta no ambiente urbano, no período de 1990 a 2019. A quantidade de artigos de pesquisa teve um crescimento notável no período de 30 anos considerado, de forma que em 1990 havia apenas 2 artigos publicados e em 2019 foi alcançado o patamar de 182 publicações.

Foram encontrados 1.067 artigos de pesquisa, sendo que 534 destes artigos estão concentrados em 26 periódicos. Ao se avaliar os títulos dos periódicos de maior destaque foi observado que os estudos sobre o uso da bicicleta em meio urbano tendem a integrar o planejamento de transportes com políticas públicas de saúde, prevenção de acidentes e sustentabilidade. 
Quanto aos autores que mais se destacaram em produção de artigos, foi percebido que estes pesquisadores estão vinculados às instituições dos seguintes países: Bélgica, Estados Unidos, Austrália, Canadá e China. As Análises no Software VOS Viewer demonstraram que a maior parcela das palavras-chave está relacionada ao Transporte Ativo, Saúde e Planejamento Urbano.

Nas análises de acoplamento bibliográfico entre países, realizadas no mesmo software, foi percebido que países como Estados Unidos, Austrália, Canadá, Bélgica e China figuram entre aqueles que apresentam o maior número de referências em comum.

Cabe salientar que não foram verificadas duplicidades de informações, ocorridas em razão dos erros nas grafias das instituições. Apesar disso, este tratamento de dados é fortemente recomendado, especialmente em estudos bibliométricos. Infere-se que este processamento adicional poderá fornecer um panorama mais detalhado, aprimorar a qualidade dos resultados obtidos e melhorar a discussão presente neste trabalho. Não obstante, o presente trabalho permitiu observar nuances do cenário mundial de pesquisas sobre práticas ciclísticas em cidades, no período de 1990 a 2019, bem como inferir quais são as perspectivas futuras de publicações para o tema.

Para trabalhos futuros, é recomendada uma investigação, de forma mais detalhada, sobre fatores que levaram ao crescimento significativo de artigos na área ao longo dos anos. Além disso, é sugerido aprofundar a discussão acerca dos estudos que integram simultaneamente os temas de planejamento de transportes, prevenção de acidentes, saúde e sustentabilidade.

\section{Agradecimentos}

O presente trabalho foi realizado com apoio da Coordenação de Aperfeiçoamento de Pessoal de Nível Superior Brasil (CAPES) - pela bolsa de mestrado (Código de Financiamento 001) de Cláudia Sabrina Pereira Lopes. Agradecemos ainda a CAPES pelo acesso ao Portal de Periódicos. Ao CNPq pela concessão de Bolsa Produtividade em Pesquisa a Antonio Conceição Paranhos Filho (PQ-1D - CNPq Processo 305013/2018-1). Ao CNPq pela bolsa PNPD a Eliane Guaraldo. Os autores também agradecem ao PGRN-UFMS (Programa de Pós-Graduação em Recursos Naturais), da Faculdade de Engenharias, Arquitetura e Urbanismo (FAENG) da Fundação Universidade Federal de Mato Grosso do Sul (UFMS).

\section{Referências}

Berrigan, D., Troiano, R. P., McNeel, T., DiSogra, C., \& Ballard-Barbash, R. (2006). Active transportation increases adherence to activity recommendations. American journal of preventive medicine, 31(3), 210-216.

Cobo, M. J., López-Herrera, A. G., Herrera-Viedma, E., \& Herrera, F. (2011). Science mapping software tools: Review, analysis, and cooperative study among tools. Journal of the American Society for information Science and Technology, 62(7), 1382-1402.

De Geus, B., De Smet, S., Nijs, J., \& Meeusen, R. (2007). Determining the intensity and energy expenditure during commuter cycling. British Journal of Sports Medicine, 41(1), 8-12.

Dill, J. (2009). Bicycling for transportation and health: the role of infrastructure. Journal of public health policy, 30(1), S95-S110.

Farrukh, M., Meng, F., Wu, Y., \& Nawaz, K. (2020). Twenty-eight years of business strategy and the environment research: A bibliometric analysis. Business Strategy and the Environment, 29(6), 2572-2582.

Gatersleben, B., \& Appleton, K. M. (2007). Contemplating cycling to work: Attitudes and perceptions in different stages of change. Transportation Research Part A: Policy and Practice, 41(4), 302-312.

Glińska, E., \& Siemieniako, D. (2018). Binge drinking in relation to services-bibliometric analysis of scientific research directions. Engineering Management in Production and Services, 10(1).

Götschi, T., Castro, A., Deforth, M., Miranda-Moreno, L., \& Zangenehpour, S. (2018). Towards a comprehensive safety evaluation of cycling infrastructure including objective and subjective measures. Journal of Transport \& Health, 8, 44-54.

Haskell, W. L. (2004). General dose response issues concerning physical activity and health. Health enhancing physical activity, 6, $149-168$. 
Research, Society and Development, v. 10, n. 11, e283101119616, 2021 (CC BY 4.0) | ISSN 2525-3409 | DOI: http://dx.doi.org/10.33448/rsd-v10i11.19616

Jacso, P. (2006). Deflated, inflated and phantom citation counts. Online information review,30(3), $297-309$.

Kaur, A., \& Sood, S. K. (2019). Analytical mapping of research on disaster management, types and role of ICT during 2011-2018. Environmental Hazards, $18(3), 266-285$

Kessler, M. M. (1963). Bibliographic coupling between scientific papers. American documentation, 14(1), 10-25.

Ma, L., \& Dill, J. (2015). Associations between the objective and perceived built environment and bicycling for transportation. Journal of Transport \& Health, 2(2), 248-255.

Milaré, G., da Silva, N. M., \& Paranhos Filho, A. C. (2016). Cenário do uso de software livre em Sistemas de Informações Geográficas (SIG) no Brasil. Anuário do Instituto de Geociências - UFRJ, 39, 111-5.

Meho, L. I., \& Rogers, Y. (2008). Citation counting, citation ranking, and h-index of human-computer interaction researchers: a comparison of Scopus and Web of Science. Journal of the American Society for Information Science and Technology, 59(11), 1711-1726.

Moore, S. C., Lee, I. M., Weiderpass, E., Campbell, P. T., Sampson, J. N., Kitahara, C. M., Keadle, S.K., Arem, H., De Gonzalez, A.B., Hartge, P., Adami, H., Blair, C. K., Borch, K. B., Boyd, E., Check, D.P., Fournier, A., Freedman, N. D., Gunter, M., Johannson, M., Khaw, K., Linet, M. S., Orsini, N., Park, Y., Riboli, E., Robien, K., Schairer, C., Sesso, H., Spriggs, M., Dusen, R. V., Wolk, A., Matthews, C. E., \& Patel, A. V. (2016). Association of leisure-time physical activity with risk of 26 types of cancer in 1.44 million adults. JAMA internal medicine, 176(6), 816-825.

Norris, M., \& Oppenheim, C. (2007). Comparing alternatives to the Web of Science for coverage of the social sciences' literature. Journal of informetrics, 1(2), 161-169.

Nocon, M., Hiemann, T., Müller-Riemenschneider, F., Thalau, F., Roll, S., \& Willich, S. N. (2008). Association of physical activity with all-cause and cardiovascular mortality: a systematic review and meta-analysis. European Journal of Preventive Cardiology, 15(3), $239-246$.

Sallis, J. F., Frank, L. D., Saelens, B. E., \& Kraft, M. K. (2004). Active transportation and physical activity: opportunities for collaboration on transportation and public health research. Transportation research part A: policy and Practice, 38(4), 249-268.

Titze, S., Stronegger, W. J., Janschitz, S., \& Oja, P. (2008). Association of built-environment, social-environment and personal factors with bicycling as a mode of transportation among Austrian city dwellers. Preventive medicine, 47(3), 252-259.

Van Eck, N. J., \& Waltman, L. (2010). Software survey: VOSviewer, a computer program for bibliometric mapping. Scientometrics, 84(2), 523-538.

Winters, M., Brauer, M., Setton, E. M., \& Teschke, K. (2013). Mapping bikeability: a spatial tool to support sustainable travel. Environment and Planning B: Planning and Design, 40(5), 865-883.

Wang, Y., Ao, Y., Zhang, Y., Liu, Y., Zhao, L., \& Chen, Y. (2019). Impact of the built environment and bicycling psychological factors on the acceptable bicycling distance of rural residents. Sustainability, 11(16), 4404.

Wang, Z., Han, Q., \& de Vries, B. (2019). Land use/land cover and accessibility: Implications of the correlations for land use and transport planning. Applied Spatial Analysis and Policy, 12(4), 923-940.

Yang, L., Chen, Z., Liu, T., Gong, Z., Yu, Y., \& Wang, J. (2013). Global trends of solid waste research from 1997 to 2011 by using bibliometric analysis. Scientometrics, 96(1), 133-146.

Yi, H., \& Xi, Z. (2008). Trends of DDT research during the period of 1991 to 2005. Scientometrics, 75(1), $111-122$. 